Bundesgesundheitsbl $2020 \cdot 63: 561-569$ https://doi.org/10.1007/s00103-020-03127-6 Online publiziert: 7. April 2020

(c) Der/die Autor(en) 2020

Benno Brinkhaus · Miriam Ortiz • Joanna Dietzel · Stefan Willich

Institut für Sozialmedizin, Epidemiologie und Gesundheitsökonomie, Charité - Universitätsmedizin Berlin, Berlin, Deutschland

\title{
Akupunktur bei Schmerzerkrankungen und Allergien - von der klinischen Erfahrung zur Evidenz
}

Manipulation der Nadel) hinausgehen, denn nur in einer sham-kontrollierten Studie fand sich ein signifikanter Effekt der Akupunktur [4, 6, 7]. Dennoch wurde die Akupunktur als Kassenleistung bei Patienten mit chronischen Gonarthroseschmerzen und Lendenwirbelsäulen(LWS-)Schmerzen anerkannt [8], weil sowohl die Akupunktur als auch die Sham-Akupunktur wirksamer war als die bisherige Standardtherapie mit einer Kombination aus Physiotherapiemaßnahmen und analgetischer Pharmakotherapie (•Tab. 1). Nach Beendigung der Modellvorhaben wurde im Jahr 2005 die "Zusatzbezeichnung Akupunktur“ für Fachärztinnen und -ärzte von der Bundesärztekammer geschaffen, die u. a. eine 200 Zeitstunden umfassende Ausbildung in Akupunktur und ein positives Prüfungsgespräch vorweisen können.

Die Wirksamkeit von Akupunktur bei chronischen Schmerzerkrankungen wurde auch nach den Modellvorhaben weiterhin kritisch diskutiert, insbesondere aufgrund des z.T. fehlenden spezifischen Effektes, und es wurde auf die Nebenwirkungen und Komplikationen von Akupunktur hingewiesen [9]. In den letzten Jahren ist eine Vielzahl von neuen Studien und Reviews veröffentlicht worden, die eine aktualisierte Bewertung der Akupunktur zulassen.

Ziel dieses Übersichtsartikels ist es, (1) die Akupunktur als Therapieverfahren vorzustellen, (2) die aktuelle Evidenz von Akupunktur bei Schmerzen und allergischen Erkrankungen aus klinischen Studien darzustellen und (3) die prakti- sche Umsetzung in Fortbildung und Anwendung in Deutschland aufzuzeigen.

\section{Akupunktur - Definition, Formen und Anwendung in Deutschland}

\section{Akupunktur - Definition}

Akupunktur (chinesisch „zhenjiu“, Akupunktur-Moxibustion-Therapie) ist eine wichtige Therapieform der Traditionellen Chinesischen und ostasiatischen Medizin $[10,11]$. Erstmals im 2. Jahrhundert vor unserer Zeitrechnung nach Funden von Texten im Mawangdui-Grab (Provinz Henan/China) schriftlich erwähnt [12], hat sich die Akupunktur im Lauf der Jahrhunderte in verschiedenen Formen weiterentwickelt [13]. Akupunktur (lat. , acus“ = Nadel, ,pungere“ = stechen) kann definiert werden als Stimulation mit verschiedenen Techniken (wie z.B. Nadeln, Moxibustion, manuellem Druck, Gleich- und Wechselstrom) von definierten Punkten des Körpers in therapeutischer Absicht [11, 13, 14]. Akupunktur wird in der chinesischen Medizin der „äußeren Therapie“ (Wai Zhi) zugeordnet - als Gegenstück zur „inneren Therapie" (Nei Zhi), unter die die Anwendung von Arzneimitteln fällt [11]. Den Grundlagen der chinesischen Medizin folgend soll durch die Akupunktur unter anderem ein Zugang zum energetischen Fließsystem des Körpers ermöglicht werden, um z. B. Blockaden zu lösen und überschüssige Energie abzuleiten [11]. Trotz der kulturellen Fremdheit Hautpenetration, oberflächlich, ohne 
Tab. 1 Wirksamkeit und Kosteneffektivität von Akupunktur bei 4 chronischen Schmerzerkrankungen. Wirksamkeit im Vergleich mit Routinebehandlung, Standardbehandlung und Sham-Akupunktur. Ergebnisse der drei Modellvorhaben zur Akupunktur 2000-2005 [4-6]. (Adaptiert nach Cummings [63])

\begin{tabular}{|c|c|c|c|c|}
\hline Diagnose & $\begin{array}{l}\text { Akupunktur } \\
\text { vs. Routinebe- } \\
\text { handlung }\end{array}$ & $\begin{array}{l}\text { Akupunktur vs. } \\
\text { Standardbe- } \\
\text { handlung }\end{array}$ & $\begin{array}{l}\text { Akupunktur } \\
\text { vs. Sham-Aku- } \\
\text { punktur }\end{array}$ & $\begin{array}{l}\text { Kostenzunahme/ } \\
\text { Kosteneffektivität }\end{array}$ \\
\hline Migräne & + & - & - & $+/+$ \\
\hline $\begin{array}{l}\text { Spannungskopf- } \\
\text { schmerzen }\end{array}$ & + & - & - & $+/+$ \\
\hline $\begin{array}{l}\text { Schmerzen an der } \\
\text { Lendenwirbel- } \\
\text { säule }\end{array}$ & + & + & - & $+/+$ \\
\hline $\begin{array}{l}\text { Gonarthrose- } \\
\text { schmerzen }\end{array}$ & + & + & + & $+/+$ \\
\hline \multicolumn{5}{|c|}{$\begin{array}{l}+=\text { wirksam, }-=\text { unwirksam. Routinebehandlung: Behandlung in der Routineversorgung, Stan- } \\
\text { dardbehandlung: Behandlung nach Leitlinien, Sham-Akupunktur: Akupunktur oberflächlich an } \\
\text { Nichtakupunkturpunkten, ohne Manipulation der Nadeln }\end{array}$} \\
\hline
\end{tabular}

der Theorie und der Therapiemethode hat sich die Akupunktur im Lauf der Jahrhunderte von einer kulturellen $\mathrm{Ku}$ riosität zu einer Therapieform entwickelt, die bei weiten Teilen der Bevölkerung in den westlichen Industrienationen Akzeptanz findet. In den letzten Jahrzehnten praktizieren zunehmend Ärztinnen und Ärzte Akupunktur unter gleichzeitiger Ablehnung des traditionellen Konzepts der chinesischen Medizin, welche als metaphysisch, antiquiert und überkommen angesehen wird. Nach einem physiologisch orientierten Ansatz werden die Akupunkturpunkte in Zusammenhang unter anderem mit myofaszialen Triggerpunkten, dem autonomen und zentralen Nervensystem und der segmentalen Zuordnung der Beschwerden ausgewählt und verwendet [15].

\section{Akupunktur - Formen}

Bei der Akupunktur werden verschiedene Formen unterschieden. Bei der Körperakupunktur werden meist feine Nadeln in definierte Punkte an der Körperoberfläche, sogenannte Akupunkturpunkte, eingestochen. Die Stimulation von Akupunkturpunkten mit Hitze durch das Abbrennen von Pflanzenextrakten aus Artemisia vulgaris (Beifußkraut) wird „Moxibustion“ genannt. Darüber hinaus kommen eine manuelle Massage von Akupunkturpunkten (Akupressur) sowie die Stimulation mit Gleich- oder Wechselstrom und La- serlicht zum Einsatz [16]. Neben der Körperakupunktur wurden spezielle Akupunkturformen, wie beispielsweise die Ohr-, Schädel- und Handakupunktur, entwickelt [17, 18].

Die chinesische Medizin definiert 361 klassische Punkte auf 14 Hauptleitbahnen („Meridianen“), die nach der Theorie der chinesischen Medizin den Körper vor allem an der Oberfläche überziehen und miteinander verbunden sind [19]. Im Verlauf der letzten beiden Jahrtausende nahm die Anzahl von Leitbahnen [10,20] und Akupunkturpunkten zu. In der täglichen Praxis wird schätzungsweise ein Repertoire von ca. 100 bis 150 Akupunkturpunkten von durchschnittlich ausgebildeten Therapeutinnen und Therapeuten werden nach der Theorie der chinesischen Medizin hinsichtlich Lokalisation, Stichtechnik, Möglichkeit der Moxibustion, Qualität des Punktes und therapeutischer Wirkung definiert [11].

In der Regel werden bei einer Körperakupunktur zwischen 1 und $25 \mathrm{Aku}-$ punkturnadeln eingesetzt, die im Verlauf eines Therapiezyklus nach individueller Diagnose variiert werden können [13]. Ein Therapiezyklus besteht bei chronischen Erkrankungen in der Regel aus 10 bis 12 Akupunktursitzungen über 3 bis 12 Wochen [13]. Häufig werden von Akupunkturärztinnen und -ärzten auch andere chinesische oder komplementärmedizinische Therapieverfahren, wie z. B. Schröpfen, Massage und Arzneieingesetzt [10]. Die Akupunkturpunkte mitteltherapie, in Kombination mit der Akupunktur eingesetzt.

\section{Akupunktur - Anwendung in Deutschland}

Akupunktur wird in Deutschland von Ärztinnen und Ärzten, Heilpraktikerinnen und Heilpraktikern und Hebammen praktiziert. In Deutschland praktizieren nach einer Publikation aus dem Jahr 2002 zwischen 30.000 bis 40.000 Ärztinnen und Ärzte Akupunktur [21], im Jahr 2018 hatten 14.648 Ärztinnen und Ärzte nach einer Anfrage bei der kassenärztlichen Bundesvereinigung eine Zusatzbezeichnung. Im Rahmen einer Befragung aus dem Jahr 1995 bei 800 Ärztinnen und Ärzten gaben $80 \%$ der Befragten an, Kenntnisse von Akupunktur zu haben, $46 \%$ beschäftigten sich mit Akupunktur. Akupunktur wird seit 1996 von der Privaten Krankenversicherung im Rahmen v.a. der Schmerztherapie erstattet, seit 2005 ist sie bei chronischem Knieschmerz und Rückenschmerzen im Katalog der Gesetzlichen Krankenversicherung aufgeführt. Die meisten Akupunkturärztinnen und -ärzte werden in Deutschland von insgesamt mehr als zehn Akupunkturgesellschaften ausgebildet, nur ein kleiner Teil erhält teilweise oder die ganze Ausbildung in China. In Deutschland setzt das Erlangen der Zusatzbezeichnung Akupunktur eine 200-stündige Weiterbildung voraus. Vor Einführung der Zusatzbezeichnung durch den Ärztetag wurde die Akupunkturausbildung in A-Diplom (140 h) und B-Diplom $(350 \mathrm{~h})$ unterteilt. In einigen Gesellschaften, wie z.B. der Internationalen Gesellschaft für Chinesische Medizin, gibt es mittlerweile ein Chinesisches Master-Diplom (1000 h). Darüber hinaus gibt es an zwei Universitäten (TU München und UKE Hamburg) seit wenigen Jahren eine postgraduelle Ausbildung in chinesischer Medizin für Ärzte. Wie viele Heilpraktiker und Hebammen in Deutschland Akupunktur praktizieren, ist derzeit unklar; Schätzungen gehen von ca. 10.000 Heilpraktikern und -praktikerinnen aus. 


\section{Akupunktur in klinischen Studien}

Die verstärkte wissenschaftliche Erforschung der Akupunktur begann nach dem bahnbrechenden Artikel einer Konsensusgruppe aus dem US-amerikanischen National Institut of Health (NIH) im Jahr 1998, in dem eine Zunahme der Inanspruchnahme von Akupunktur festgestellt wurde, gleichzeitig ein Mangel an qualitativ hochwertigen Studien zur Wirksamkeit [3]. Bis zu diesem Zeitpunkt gab es eine positive Evidenz nur für postoperative und chemotherapieinduzierte Übelkeit bzw. Erbrechen sowie postoperativen Zahnschmerz. Es wurden andere Indikationen aufgelistet, bei denen Akupunktur nach der damals vorliegenden wissenschaftlichen Literatur möglicherweise wirksam sein konnte: Sucht und Abhängigkeit, Apoplexrehabilitation, Kopfschmerz, Dysmenorrhö, Tennisellenbogen, Fibromyalgie, myofaszialer Schmerz, Gonarthrose, LWSSchmerzen, Karpaltunnelsyndrom und Asthma.

Die Autoren forderten qualitativ hochwertige klinische Studien, die sich mit der Wirksamkeit unter Routinebedingungen, der spezifischen Wirksamkeit, der Therapiesicherheit und den Kosten von Akupunktur auseinandersetzen. Etwa zur gleichen Zeit wurde nach Analyse der bisher in China publizierten Studien bekannt, dass diese nicht den methodischen Ansprüchen für qualitativ hochwertige Forschung genügten, in der Regel positiver waren als die westlichen Studien und dass ein erheblicher Publikationsbias vorlag [22].

Seit dem Artikel des NIH im Jahr 1998 ist die Anzahl von Publikationen wissenschaftlicher Artikel zur Akupunktur exponentiell gestiegen: Wurden bis 1999 etwa 3000 Artikel zur Akupunktur publiziert, waren es 20 Jahre später schon etwa 30.200 (• Abb. 1). Auch die Qualität der Studien nahm, zunächst in den westlichen Industrienationen wie Deutschland, Großbritannien, Spanien, Schweden und den USA deutlich zu. In den letzten Jahren hat sich die Studienqualität in China ebenfalls deutlich verbessert (siehe Akupunktur bei anderen Erkrankungen).

Bundesgesundheitsbl 2020 · 63:561-569 https://doi.org/10.1007/s00103-020-03127-6

(c) Der/die Autor(en) 2020

B. Brinkhaus · M. Ortiz · J. Dietzel · S. Willich

\section{Akupunktur bei Schmerzerkrankungen und Allergien - von der klinischen Erfahrung zur Evidenz}

\section{Zusammenfassung}

Hintergrund. Die Akupunktur ist ein Therapieverfahren der Traditionellen Chinesischen Medizin, das inzwischen auch in der westlichen Welt häufig eingesetzt wird. In den letzten beiden Jahrzehnten wurde es intensiv wissenschaftlich untersucht. Ziel der Arbeit. Darstellung von Wirksamkeit, Therapiesicherheit und gesundheitsökonomischer Relevanz der Akupunktur bei chronischen Schmerzerkrankungen und Allergien.

Material und Methoden. Im Rahmen einer Übersichtsarbeit (narratives Review) wird die relevante Literatur ausgesucht, vorgestellt und interpretiert.

Ergebnisse. Akupunktur hat sich bei vielen chronischen Schmerzerkrankungen unter Routinebedingungen als wirksam erwiesen, so z. B. bei Kniegelenksschmerzen aufgrund von Arthrose, bei Migräne, Spannungskopfschmerz, chronischen Schmerzen der Lendenund Halswirbelsäule, Schulterschmerzen, Dysmenorrhö und Fibromyalgie. Außerdem zeigt sich bei den meisten Schmerzerkrankungen eine spezifische Wirksamkeit. Auch bei Patienten mit allergischen Erkrankungen ist Akupunktur wirksam. Akupunktur hat sich in den großen klinischen Studien als relativ sicheres Therapieverfahren erwiesen, allerdings kommen in seltenen Fällen auch schwere Komplikationen vor (z. B. Pneumothoraxfälle, Hepatitisinfektionen). In der Regel ist Akupunktur mit zusätzlichen Therapiekosten verbunden, gilt allerdings bei Schmerzerkrankungen als kosteneffektive Therapiestrategie.

Diskussion. Als nichtpharmakologischem Verfahren kommt Akupunktur eine wachsende Bedeutung bei der Behandlung von chronischen Schmerzerkrankungen und Allergien zu. Sie wird daher zunehmend in aktuelle Therapieleitlinien aufgenommen. In weiteren Studien sollte ihre Wirksamkeit bei weiteren Indikationen bestimmt werden. Die Wirkmechanismen sollten untersucht werden.

\section{Schlüsselwörter}

Akupunktur · Komplementärmedizin . Chronische Schmerzen · Allergie · Traditionelle Chinesische Medizin

\section{Acupuncture for pain and allergic rhinitis-from clinical experience to evidence}

\section{Abstract}

Background. Acupuncture is a traditional Chinese medicine therapy method that is also frequently used in the western world. It has been the subject of intensive scientific research over the past two decades.

Aim of the work. To describe the efficacy, therapy safety, and health economic relevance of acupuncture for chronic pain disorders and allergic diseases.

Material and methods. In the context of a review (narrative review) the relevant literature was selected, presented, and interpreted.

Results. Acupuncture is effective in chronic pain disorders such as chronic knee joint pain in osteoarthritis, migraine, tension headache, chronic lumbar spine pain, chronic cervical spine pain, shoulder pain, dysmenorrhea, and fibromyalgia under routine conditions. In addition, specific efficacy is shown in most chronic pain indications. Acupuncture is also effective in patients with allergic diseases.
In large clinical studies, acupuncture has proven to be a relatively safe therapy, but severe complications (e.g. pneumothorax cases, hepatitis B) also occur in rare instances. Acupuncture is usually associated with higher therapy costs, but is considered a cost-effective treatment strategy for pain disorders.

Discussion. As a nonpharmacological procedure, acupuncture is becoming increasingly important in pain and allergy therapy and is therefore increasingly included in current guidelines. In further studies, the efficacy of acupuncture should be determined in other indications and the mechanisms of action of this therapy method should be investigated.

\section{Keywords}

Acupuncture - Complementary medicine . Chronic pain · Allergy · Traditional chinese medicine 


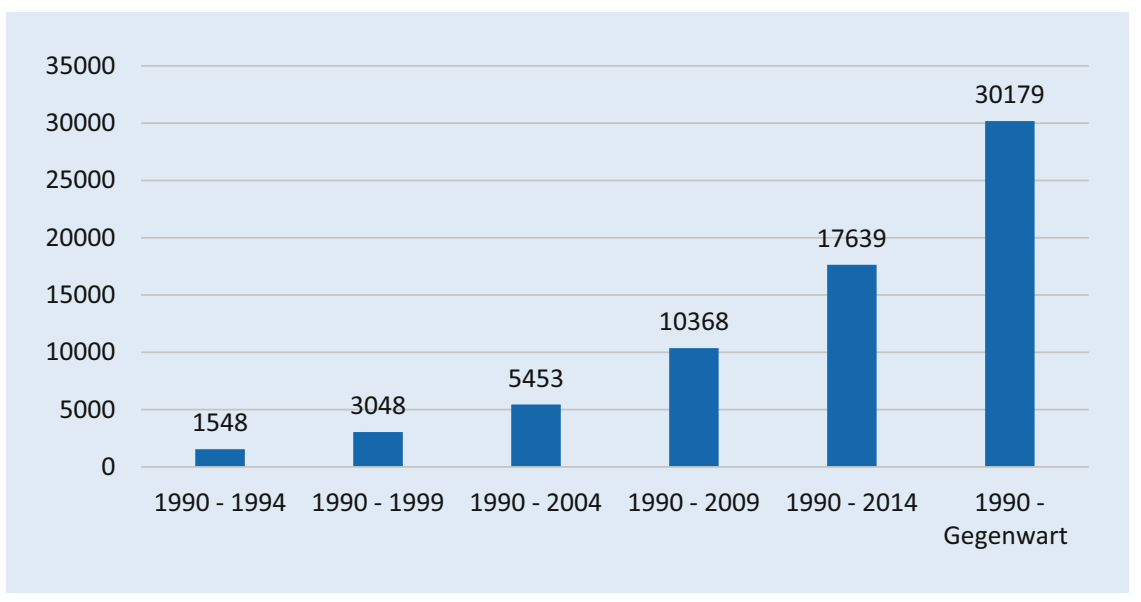

Abb. 1 ॥ Zunahme der Anzahl von Publikationen zu Akupunkturvon 1990 bis Mai 2019. (Datenquelle: PubMed)

Im Jahr 2007 entwickelten zwei Forschergruppen für Verfahren der komplementären Medizin, wie z.B. für die Akupunktur, die sogenannte Reverse Research Strategy: Anders als bei der üblichen pharmakologischen Forschung sieht diese Strategie vor, bei traditionellen Therapieverfahren wie der Akupunktur, für die es durch die z.T. jahrhundertelange Anwendungstradition ein großes Erfahrungswissen gibt, zunächst die Therapiesicherheit und dann die Wirksamkeit unter Routinebedingungen $\mathrm{zu}$ untersuchen, bevor die spezifische Wirksamkeit und der Wirkmechanismus Gegenstand der Forschung sind [23, 24].

Diese Forschungsstrategie wurde auch in die Research Roadmap für Komplementärmedizin im von der EU geförderten CAMbrella Projekt aufgenommen [25] und bildet die Grundlage für die weitere Erforschung der Akupunktur. Im Weiteren wird auf die aktuelle Evidenzlage der Akupunktur bei Schmerzerkrankungen, Allergien und weiteren Erkrankungen eingegangen.

\section{Akupunktur bei chronischen Schmerzen}

Die Modellvorhaben zur Akupunktur in Deutschland zeigten die Wirksamkeit bei den untersuchten vier chronischen Schmerzerkrankungen Migräne, Spannungskopfschmerz, Gonarthrose und LWS-Schmerzen unter Routinebedingungen ([4, 6]; - Tab. 1). Allerdings fand sich nur in einer der großen acht randomisierten Acupuncture Randomised Trials (ART) und German-Acupuncture-Trials-(GERAC-)Studien, die im Rahmen der Modellvorhaben in Deutschland durchgeführt wurden, ein eindeutiger Hinweis auf eine spezifische Wirksamkeit, was von vielen Kritikern [9] bemängelt wurde: Ist die Akupunktur lediglich eine Placebotherapie?

Dies war auch der Hintergrund, vor dem die mit internationalen Experten besetzte und in den USA primär angesiedelte Acupuncture Trialist's Cooperation (ATC-Gruppe) gegründet wurde mit dem Ziel, im Rahmen einer großen Metaanalyse von Patientendaten die Therapieeffekte von Akupunktur bei chronischen Schmerzerkrankungen in den zur Verfügung stehenden qualitativ hochwertigen Studien zu untersuchen. Die Ergebnisse dieser Studien wurden in sogenannten Effektgrößen angegeben, wobei eine Effektgröße bis 0,40 als „kein therapeutischer Effekt" interpretiert wurde und Werte von 0,4 bis 0,6 als „mittlerer“ bzw. größer 0,6 als „großer therapeutischer Effekt“. Die Ergebnisse von den 29 eingeschlossenen Studien bei 17.922 Patientinnen und Patienten zeigten, dass Akupunktur bei allen Schmerzindikationen in der Routineversorgung mit mittleren Effektgrößen von 0,42-0,57 wirksam ist [26]. Es zeigten sich auch in allen Studien signifikante Effekte von Akupunktur gegenüber Sham-Akupunktur bei allen Schmerzerkrankungen; allerdings wurden bei diesem Vergleich nur kleine Effektgrößen von 0,15-0,25 ermittelt.
Auch ein Update der genannten Studie im Jahr 2018 zeigte unter dem Einschluss von 39 Studien mit 20.827 aufgenommenen Patientinnen und Patienten ein nahezu identisches Bild mit einer kleinen Effektgröße von 0,2, mit einem signifikanten Unterschied von Akupunktur vs. Sham-Akupunktur bei allen Schmerzindikationen (alle $p<0,001$ ) und einer mittleren Effektgröße von 0,5 Akupunktur in der Routineversorgung [27]. Dabei waren die Effektgrößen der Akupunktur in der Routineversorgung bei den verschiedenen Schmerzerkrankungen sehr unterschiedlich: Arthroseschmerzen: 0,63 (95\%-Konfidenzintervall (KI) 0,56-0,69), muskuloskelettale Schmerzen: 0,54 (95\%-KI 0,50-0,57), chronische Kopfschmerzen: 0,44 (95\%KI 0,39-0,48). Auch beim Vergleich der Akupunktur zur Sham-Akupunktur fanden sich je nach Diagnose Unterschiede in den Effektgrößen: Arthroseschmerz: 0,24 (95\%-KI 0,17-0,31), muskuloskelettaler Schmerz: 0,30 (95\%KI 0,21-0,38), Schulterschmerz: 0,57 (95\%-KI 0,44-0,69), chronischer Kopfschmerz: 0,16 (95\%-KI 0,08-0,25). Es fand sich auch eine eindeutige Evidenz zur Wirkdauer des Akupunktureffekts mit einem Wirkverlust von ca. $15 \%$ nach einem Jahr.

Nach 20 Jahren intensiver klinischer Forschung zeigt sich, dass die Akupunktur bei vielen untersuchten Indikationen in der Routineversorgung wirksam ist und spezifische Effekte zeigt (• Tab. 2). Aus diesem Grund wurde sie zunehmend interessanter für die Darstellung und $\mathrm{Pu}$ blikation in hochrangigen wissenschaftlichen Journalen [28]. Seit einigen Jahren wird Akupunktur, insbesondere bei chronischen Schmerzerkrankungen, auch zunehmend in medizinische Leitlinien aufgenommen, z. B. bei chronischen LWSSchmerzen [29, 30].

Für das Gesundheitssystem ist vor allem relevant, ob durch die Akupunktur Kosten reduziert werden können. Bei den gesundheitsökonomischen Analysen zeigte sich, dass die Durchführung von Akupunktur in der Regel mit mehr Kosten verbunden ist. Allerdings zeigte sich in allen Schmerzstudien, dass Akupunktur kosteneffektiv ist, d.h., dass die Patientinnen und Patienten als Gegenwert 


\begin{tabular}{|c|c|c|c|c|}
\hline Erkrankung & Autor(en) & Journal & Studien & $\begin{array}{l}\text { Schlussfolgerung } \\
\text { Effectiveness/Efficacy }\end{array}$ \\
\hline Gonarthrose & $\begin{array}{l}\text { Manheimer } \\
\text { et al. [64] }\end{array}$ & $\begin{array}{l}\text { Ann Int Med } \\
2007\end{array}$ & 11 & $+/+$ \\
\hline Migräne & $\begin{array}{l}\text { Linde et al. } \\
\text { [65] }\end{array}$ & $\begin{array}{l}\text { Cochrane } \\
\text { Database } 2016\end{array}$ & 22 & $+/+$ \\
\hline $\begin{array}{l}\text { Spannungskopf- } \\
\text { schmerz }\end{array}$ & $\begin{array}{l}\text { Linde et al. } \\
\text { [66] }\end{array}$ & $\begin{array}{l}\text { Cochrane } \\
\text { Database } 2016\end{array}$ & 11 & $+/+$ \\
\hline $\begin{array}{l}\text { Chronischer Schmerz } \\
\text { der Lendenwirbelsäule } \\
\text { (LWS) }\end{array}$ & $\begin{array}{l}\text { Furlan et al. } \\
\text { [67] }\end{array}$ & $\begin{array}{l}\text { Cochrane } \\
\text { Database } 2005\end{array}$ & 35 & $+/+$ \\
\hline $\begin{array}{l}\text { Chronischer Schmerz } \\
\text { der Halswirbelsäule } \\
\text { (HWS) }\end{array}$ & $\begin{array}{l}\text { Trinh et al. } \\
\text { [68] }\end{array}$ & $\begin{array}{l}\text { Cochrane } \\
\text { Database } 2006\end{array}$ & 10 & $+/+$ \\
\hline Reizdarmsyndrom & $\begin{array}{l}\text { Manheimer } \\
\text { et al. [69] }\end{array}$ & $\begin{array}{l}\text { Cochrane } \\
\text { Database } 2012\end{array}$ & 17 & $+/-$ \\
\hline Fibromyalgie & $\begin{array}{l}\text { Deare et al. } \\
\text { [70] }\end{array}$ & $\begin{array}{l}\text { Cochrane } \\
\text { Database } 2013\end{array}$ & 9 & $+/-$ \\
\hline Dysmenorrhö & $\begin{array}{l}\text { Smith et al. } \\
\text { [71] }\end{array}$ & $\begin{array}{l}\text { Cochrane } \\
\text { Database } 2016\end{array}$ & 42 & $+/-$ \\
\hline Koxarthrose & $\begin{array}{l}\text { Manheimer } \\
\text { et al. [72] }\end{array}$ & $\begin{array}{l}\text { Cochrane } \\
\text { Database } 2018\end{array}$ & 5 & $+/-$ \\
\hline
\end{tabular}

eine deutlich bessere Lebensqualität erhalten (gemessen in qualitätskorrigierten Lebensjahren, QALYs; [31-34]).

Sham-kontrollierte Studien bei Schmerzerkrankungen sind seit längerer Zeit bei Akupunkturforschern in der Diskussion, da die Sham-Akupunktur keinem inerten Placebo entspricht. $\mathrm{Zu}-$ dem wird immer wieder kritisch darauf hingewiesen, dass in der Akupunktur, wie bei vielen anderen komplexen Therapieverfahren, keine doppelt verblindeten Studien möglich wären, da eine bewusste oder unbewusste Beeinflussung der Patientinnen und Patienten durch die Prüfärztinnen und Prüfärzte nicht vermieden werden kann. Die Ergebnisse einer Metaanalyse von 37 Studien mit 5754 Patientinnen und Patienten zeigte, dass auch Sham-Akupunktur eine Effektgröße von $-0,45$ vs. „keine Behandlung“ hat und dass Studien mit einem größeren Effekt der Sham-Akupunktur vs. „keine Behandlung" zugleich einen signifikant kleineren Effekt $(p=0,029)$ der Akupunktur vs. Sham-Akupunktur haben [35].

Daher wird zunehmend der primäre Vergleich von Akupunktur vs. Standardtherapie oder Routinebehandlung bei Schmerzerkrankungen in klinischen Studien empfohlen. Insgesamt muss davon ausgegangen werden, dass ein eher kleinerer Teil des Akupunktureffekts durch einen spezifischen Effekt verursacht wird und ein größerer Teil durch sogenannte unspezifische bzw. Kontextfaktoren. Wie diese Faktoren durch die Verbesserung der Kommunikation bzw. Empathie gesteigert werden, konnte in einer Studie eindrucksvoll bewiesen werden [36], ebenfalls, dass ein großer Teil des Effekts durch die Erwartungshaltung maßgeblich beeinflusst wird [37].

\section{Akupunktur bei allergischen Erkrankungen}

Zwischen $27 \%$ und $46 \%$ der Patientinnen und Patienten mit saisonaler allergischer Rhinitis (SAR) nehmen komplementärmedizinische Methoden in Anspruch: Akupunktur wird bei Allergien mit einer Lebenszeitprävalenz von ca. $19 \%$ relativ häufig eingesetzt [38]. Dabei war die Evidenz von Akupunktur lange Zeit unklar. Das zeigten auch die Ergebnisse einer systematischen Übersichtsarbeit aus dem Jahr 2008, in die insgesamt 7 randomisierte Studien eingeschlossen wurden; bis auf 2 Studien hatten alle anderen eine niedrige Studienqualität [39]. Die Schlussfolgerung der Autoren war, dass die damalige Evidenz nicht ausreichte, um den Einsatz der Akupunktur bei Allergien zu unterstützen. Die Autoren empfahlen die Initiierung von qualitativ hochwertigen Studien zur Verbesserung der Evidenz.

Seit dem genannten Review sind weitere Studien publiziert worden. Im Rahmen einer großen dreiarmigen teilrandomisierten pragmatischen Multicenterstudie mit 5237 Allergiepatienten konnte gezeigt werden, dass die Gruppe der Patientinnen und Patienten, die (randomisiert oder nichtrandomisiert zugeteilt) Akupunktur in den ersten drei Monaten zusätzlich zur Routinebehandlung erhielten, nach 12 Wochen ein besseres Ergebnis erzielte als die Gruppe, die nach Randomisierung in den ersten 3 Monaten nur die Routinetherapie, aber keine Akupunktur erhielt [40]. In einer dreiarmigen randomisierten kontrollierten Multicenterstudie mit 422 Patientinnen und Patienten fanden sich Hinweise auf eine spezifische Wirksamkeit der Akupunktur bei SAR. Patientinnen und Patienten in der Akupunkturgruppe hatten nach 8 Wochen eine höhere spezifische Lebensqualität und nahmen weniger Antihistaminika als Bedarfsmedikation ein im Vergleich zu denen, die eine ShamAkupunktur oder nur Bedarfsmedikation erhalten hatten [41]. Interessanterweise fanden sich in dieser Studie auch im Folgejahr noch signifikant bessere Effekte in der Akupunkturgruppe im Vergleich zur Sham-Akupunkturgruppe, ohne dass die Patientinnen und Patienten weitere Akupunkturbehandlungen erhielten. Dies bestätigt die Erfahrungen von Therapeutinnen und Therapeuten und weist auf einen Langzeiteffekt der Akupunktur bei SAR hin.

Auch in einer weiteren randomisierten kontrollierten Studie bei 175 Patientinnen und Patienten mit SAR war die Akupunktur der Sham-Akupunktur hinsichtlich der Symptomreduktion und der Verbesserung der spezifischen Lebensqualität überlegen [42]. Ergebnisse einer koreanisch-chinesischen randomisiert kontrollierten Multicenterstudie bei 238 Patientinnen und Patienten mit per- 
sistierender allergischer Rhinitis (PAR) zeigten ebenfalls spezifische Effekte der Akupunktur bei nasalen Beschwerden im Vergleich zu einer Sham-Kontrolle; allerdings fanden sich in dieser Studie keine Unterschiede bei den nichtnasalen Beschwerden, wie z. B. Konjunktivitis [43].

In einem systematischen Review wurden die Ergebnisse von 13 RCT-Studien (publiziert bis Juni 2013) mit insgesamt 2365 Allergiepatienten zusammengefasst: Es zeigte sich ein signifikanter Unterschied der Akupunktur gegenüber den Kontrollgruppen hinsichtlich der Verbesserung der nasalen Beschwerden, der Einnahme von antiallergischer Bedarfsmedikation und dem Rückgang der IgE-Antikörper [44], nicht aber hinsichtlich der spezifischen Lebensqualität. Die Autoren kamen zu der Schlussfolgerung, dass Akupunktur eine sichere und valide Therapieoption bei Patientinnen und Patienten mit allergischer Rhinitis sein kann.

In den USA wurde die Akupunktur unter dem Statement 13 in die klinische Leitlinie für die Hals-Nasen-Ohren-(HNO-)Praxis aufgenommen [45]: Die Schlussfolgerung dieser Empfehlung ist, dass Kliniker Patienten, die an einer nichtpharmakologischen Therapie interessiert sind, entweder selber Akupunktur anbieten oder sie an einen Akupunkturtherapeuten verweisen. In der aktuellen Leitlinie der Deutschen Gesellschaft für Allergologie und klinische Immunologie wird die Akupunktur derzeit noch nicht empfohlen, auch weil es inzwischen qualitativ hochwertige Studien zur Wirksamkeit und Verträglichkeit der spezifischen Immuntherapie gibt [46], sodass dieser Therapie der Vorzug gegenüber einer symptomatischen Therapie gegeben wird.

Die Ergebnisse der Kostenanalyse in einer Studie [41] zeigten, dass Akupunktur in den ersten 4 Monaten nach Beginn der Intervention mit höheren Kosten verbunden ist. Die Patientinnen und Patienten der Akupunkturgruppe hatten jedoch auch signifikant höhere QALYs im Vergleich zur Bedarfsmedikationsgruppe, allerdings war die Akupunktur nach 4 Monaten nicht kosteneffektiv [47].

Der Wirkmechanismus von Akupunktur bei allergischer Rhinitis ist derzeit unklar und bedarf weiterer Erforschung. Eine Hypothese ist, dass Akupunktur durch ein komplexes neuroendokrinimmunologisches Netzwerk wirkt, da bekannt ist, dass Akupunktur auf verschiedenen Ebenen antiinflammatorische Effekte (z.B. antihistaminerge Effekte, Down-Regulation von proinflammatorischen Zytokinen und proinflammatorischen Neuropeptiden) in Untersuchungen aufweist [48]. Trotz der derzeitig positiven klinischen Evidenz der Akupunktur bei allergischen Erkrankungen wird diese erst dann vollständig akzeptiert sein, wenn es gelingt, mindestens eine der oben genannten Hypothesen zum spezifischen Wirkmechanismus zu bestätigen.

\section{Akupunktur bei anderen Erkrankungen}

Akupunktur wird bei einer Vielzahl von weiteren Erkrankungen eingesetzt, darunter insbesondere Erkrankungen des Bewegungsapparates, aber auch internistische Erkrankungen (z.B. koronare Herzerkrankung, Reizdarmsyndrom, chronisch entzündliche Darmerkrankungen), neurologische (Polyneuropathie, multiple Sklerose, Schlaganfall) und psychische Erkrankungen (Depression). Ziel einer australischen Arbeitsgruppe war es, sich einen Überblick über den Forschungsstand zu verschaffen. $\mathrm{Zu}$ diesem Zweck wertete sie insbesondere systematische Reviews mit Metaanalysen aus, darunter insgesamt 159 qualitativ hochwertige Cochrane-Reviews [49]. Eine positive Evidenz fand sich nach der Analyse der Daten bei den folgenden Indikationen: allergische Rhinitis, Migräneprophylaxe, Spannungskopfschmerz, Halswirbelsäulen-(HWS-) und LWSSchmerzen, Knieschmerzen, Schmerzen und Übelkeit nach einer Operation sowie chemotherapieinduzierte Übelkeit. Bei 38 anderen Indikationen lagen positive RCTs vor (u.a. ambulante Anästhesie, Menopausensyndrom, Reizdarmsyndrom, Apoplexie), bei 71 Indikationen war die Evidenz nicht ausreichend (u.a. bei Alzheimer, Tinnitus), bei 5 Indikationen gab es keinen Hinweis auf Wirksamkeit (u. a. bei Alkohol-, Nikotin- und Kokainentzug).
Im letzten Jahrzehnt wurden in China zunehmend hochwertige RCT-Studien zur Wirksamkeit von Akupunktur bei großen Patientenpopulationen mit unterschiedlichen Erkrankungen durchgeführt, u. a. eine negative Studie beim polyzystischen Ovarsyndrom [50], eine positive Studie bei schwerer funktionaler Obstipation [51], eine positive Studie bei Stressharninkontinenz [52], eine positive Studie bei klimakterischen Beschwerden mit einem klinisch nicht relevanten Effekt [53] und eine positive Studie bei stabiler Angina-Pectoris-Symptomatik [54].

\section{Studien zur Wirksamkeit von Moxibustion}

Es liegen sehr viel weniger Studien zur Moxibustion vor im Vergleich zur Akupunktur. Das liegt zum einen daran, dass sehr viel weniger Moxibustion als Akupunktur durchgeführt wird, aber auch weil sich Moxibustion aufgrund der Kombination aus der Wärme- und Geruchskomponente sehr viel schlechter verblinden lässt. Außerdem wird Moxibustion oft in Kombination mit Akupunktur angewendet und nicht als singuläres Verfahren. Bisher ist es auch nicht gelungen, ein Sham- oder Placeboverfahren für Moxibustion zu entwickeln [55]. In der Praxis wird Moxibustion über den Akupunkturpunkt Bl 67 häufig ohne Kombination mit Akupunktur bei Beckenfehllage in der späten Schwangerschaft eingesetzt. In einer italienischen RCT-Studie mit 130 Patientinnen in der 33. Schwangerschaftswoche (SSW) fand sich nach 1- bis 2-wöchiger Moxibustion eine signifikant höhere Wendungsrate im Vergleich zu der Gruppe, die nur eine Routinebehandlung erhielt [56]. Diese Ergebnisse wurden durch eine spanische dreiarmige RCT-Studie mit 409 Frauen in der 32.-35. SSW bestätigt. Hier kam es unter der Moxibustion des Punktes Bl 67 häufiger zur Wendung (58\%) als unter der Moxibustion des Punktes Mi 1 $(43 \%)$ und unter Routinebehandlung (45\%; [57]). Weitere Studien liegen z. B. zur Wirksamkeit von Moxibustion bei Herpes Zoster [58] und in Kombination mit Akupunktur bei Morbus Crohn vor [59]. 


\section{Therapiesicherheit}

Kein komplementärmedizinisches Therapieverfahren ist bezüglich der $\mathrm{Ne}$ benwirkungen so gut untersucht wie die Akupunktur. Alle bisherigen Untersuchungen zeigen, dass Akupunktur ein relativ sicheres und nebenwirkungsarmes Therapieverfahren ist, wenn es fachgerecht appliziert wird. Allerdings kann es in seltenen Fällen auch z.T. zu schweren Komplikationen kommen. In einer englischen Studie mit 9408 Patientinnen und Patienten und nach insgesamt über 30.000 Akupunkturbehandlungen, die von nichtärztlichen Therapeutinnen und Therapeuten durchgeführt wurden, wurden insgesamt $10,7 \%$ Nebenwirkungen dokumentiert. Neben Hämatomen und Blutungen traten v.a. Ermüdung $(3,6 \%)$ und verlängertes Schmerzempfinden $(1,6 \%)$ auf [60]. Autoren einer deutschen Studie fanden nach Berichten von 7050 Ärzten insgesamt 7,1\% Patienten mit Nebenwirkungen bei 760.000 Behandlungen, zumeist $\mathrm{Na}$ delschmerz $(3,3 \%)$ und Hämatome $(3,2 \% ;[61])$. Studienergebnisse einer mit 229.230 Patienten noch größeren Population, die ca. 2,2 Mio. Behandlungen erhielt, zeigten in $8,6 \%$ mindestens eine Nebenwirkung, wobei die häufigsten Nebenwirkungen Blutungen/Hämatome $(6,2 \%)$, Schmerz $(1,7 \%)$ und vegetative Symptome $(0,7 \%)$ waren [62]. In dieser Studie fanden sich auch $2 \mathrm{~Pa}$ tienten mit einem durch Akupunktur verursachten Pneumothorax (Häufigkeit 1:1 Mio. Behandlungen), der aber nicht lebensgefährlich war und nicht invasiv behandelt werden musste. Die Autoren dieser Arbeit wiesen in ihrer Zusammenfassung auf die Bedeutung einer adäquaten Aufklärung mit Einholen eines schriftlichen Einverständnisses der Patientinnen und Patienten hin und präsentierten eine Version einer solchen Patienteninformation [62]. Dieser Patienteninformation kommt eine wichtige Bedeutung zu, da zwar in seltenen Fällen, aber immer wieder von schweren Komplikationen berichtet wird, die insbesondere Organverletzungen und die Übertragung von Infektionskrankheiten betreffen; Todesfälle sind beschrieben worden [9].

\section{Fazit}

Akupunktur hat sich in den letzten 3 Jahrzehnten von einer Außenseitermethode - aus der Traditionellen Chinesischen Medizin kommend - zu einem zunehmend akzeptierten Therapieverfahren in der westlichen Welt entwickelt, insbesondere bei Schmerzerkrankungen, aber auch bei anderen Indikationen, wie z. B. bei allergischen Erkrankungen. Akupunktur ist zudem eine relativ sichere Therapiemethode. Die zusätzlichen Gesamttherapiekosten erscheinen insbesondere bei den Schmerzindikationen durch den Gewinn an Lebensqualität gerechtfertigt. Die spezifische Wirksamkeit von Akupunktur ist aktuell Gegenstand von vielen experimentellen Studien, die das komplizierte System der Wirkmechanismen untersuchen. Erst wenn die Wirkmechanismen eindeutig und nachvollziehbar identifiziert sind, wird die Akupunktur vorbehaltlos in die konventionelle Medizin integriert werden und der Weg vom Mythos zur Evidenz somit erfolgreich abgeschlossen sein.

\section{Korrespondenzadresse}

\section{Prof. Dr. Benno Brinkhaus}

Institut für Sozialmedizin, Epidemiologie und Gesundheitsökonomie, Charité Universitätsmedizin Berlin Luisenstr. 57, 10117 Berlin, Deutschland benno.brinkhaus@charite.de

Danksagung. Wir danken Frau Cree für die Bearbeitung der Literatur und Frau Sylvia Binting für die Unterstützung bei der Bearbeitung der Abbildungen und Tabellen.

Funding. Open Access funding provided by Projekt DEAL.

\section{Einhaltung ethischer Richtlinien}

Interessenkonflikt. B. Brinkhaus erklärt, dass die zweite Phase (2015-2019) seiner Stiftungsprofessur am Institut für Sozialmedizin, Epidemiologie und Gesundheitsökonomie der Charité - Universitätsmedizin Berlin von der Krankenkasse BKK24, der Immanuel Diakonie Group, vom Kneipp Bund e. V., von der Software AG-Stiftung, vom Komitee Forschung Naturmedizin und von der Stadt Bad Wörishofen gefördert wurde. Er erhielt Fahrtkosten und/oder Honorare von verschiedenen komplementärmedizinischen Gesellschaften für Vorträge und sein Team Naturheilkunde wurde von der Firma China Purmed GmbH von 2005-2008 unterstützt. M. Ortiz erklärt, dass sie Vortragshonorare von der Deutschen Ärztegesellschaft für Akupunktur e. V. (DÄGfA) erhalten hat. J. Dietzel erklärt, dass sie ein Habilitationsstipendium von der „Karl und Veronica Carstens-Stiftung" zur Durchführung klinischer Forschung im Bereich Akupunktur erhalten hat. S. Willich gibt an, dass kein Interessenkonflikt besteht. Von keinem der Autoren wurden Kurse zur Akupunktur bei den Ärztegesellschaften angeboten.

Für diesen Beitrag wurden von den Autoren keine Studien an Menschen oder Tieren durchgeführt. Für die aufgeführten Studien gelten die jeweils dort angegebenen ethischen Richtlinien.

Open Access Dieser Artikel wird unter der Creative Commons Namensnennung 4.0 International Lizenz veröffentlicht, welche die Nutzung, Vervielfältigung Bearbeitung, Verbreitung und Wiedergabe in jeglichem Medium und Format erlaubt, sofern Sie den/die ursprünglichen Autor(en) und die Quelle ordnungsgemäß nennen, einen Link zur Creative Commons Lizenz beifügen und angeben, ob Änderungen vorgenommen wurden.

Die in diesem Artikel enthaltenen Bilder und sonstiges Drittmaterial unterliegen ebenfalls der genannten Creative Commons Lizenz, sofern sich aus der Abbildungslegende nichts anderes ergibt. Sofern das betreffende Material nicht unter der genannten Creative Commons Lizenz steht und die betreffende Handlung nicht nach gesetzlichen Vorschriften erlaubt ist, ist für die oben aufgeführten Weiterverwendungen des Materials die Einwilligung des jeweiligen Rechteinhabers einzuholen.

Weitere Details zur Lizenz entnehmen Sie bitte der Lizenzinformation auf http://creativecommons.org/ licenses/by/4.0/deed.de.

\section{Literatur}

1. Eardley S, Bishop FL, Prescott P et al (2012) A systematic literature review of complementary and alternative medicine prevalence in EU. Forsch Komplementarmed 19(Suppl 2):18-28

2. Hartel U, Volger E (2004) Use and acceptance of classical natural and alternative medicine in Germany - findings of a representative populationbased survey. Forsch Komplementarmed Klass Naturheilkd 11:327-334

3. Anonymous (1998) NIH Consensus Conference. Acupuncture. JAMA 280:1518-1524

4. Witt CM, Brinkhaus B, Jena $S$ et al (2006) Wirksamkeit, Sicherheit und Wirtschaftlichkeit der Akupunktur - Ein Modellvorhaben mit der Techniker Krankenkasse. Dtsch Ärztebl Int 103:196-203

5. Endres HG, Victor N, Haake Met al (2007) Akupunktur bei chronischen Knie- und Rückenschmerzen. Dtsch Ärztebl Int 104:123-130

6. Melchart D, Streng A, Hoppe A et al (2006) Akupunktur bei chronischen Schmerzen: Ergebnisse aus dem Modellvorhaben der Ersatzkassen. Dtsch Ärztebl Int 103:187-195

7. WittC, Brinkhaus B, Jena Setal (2005) Acupuncture in patients with osteoarthritis of the knee: a randomised trial. Lancet 366:136-143

8. Gemeinsamer Bundesausschuss (2006) Akupunktur zur Behandlung von Rücken- und Knieschmerzen wird Kassenleistung. https://www.g-ba.de/ presse/pressemitteilungen/83/. Zugegriffen: 17. Jan. 2020 
9. Ernst E, Lee MS, Choi TY (2011) Acupuncture: Does it alleviate pain and are there serious risks? A review of reviews. Pain 152:755-764

10. Kaptchuk TJ (2002) Acupuncture: theory, efficacy, and practice. Ann Intern Med 136:374-383

11. Porkert M, Hempen CH (1985) Systematische Akupunktur. Urban \& Schwarzenberg, München

12. Despeux C (2000) Chinesische Medizin. Chin Med 15:145-152

13. Vickers A, Zollman C (1999) ABC of complementary medicine. Acupuncture. BMJ 319:973-976

14. NasirLS (2002) Acupuncture.Prim Care 29:393-405

15. Filshie J, White A (1998) Medical acupuncture: a Western scientific approach. Churchill Livingstone, London

16. Whittaker P (2004) Laser acupuncture: past, present, and future. Lasers Med Sci 19:69-80

17. Hsu E (1996) Innovations in acumoxa: acupuncture analgesia, scalp and ear acupuncture in the People's Republic ofChina. Soc Sci Med 42:421-430

18. Angermaier M (2001) Leitfaden Ohrakupunktur. Urban \& Fischer, München

19. Deadman P, Baker K, Al-Khafaji M (1998) A manual of acupuncture. Eastland Press, Seattle

20. Kirschbaum B (2000) Die 8 außerordentlichen Gefäße in der traditionellen chinesischen Medizin. Medizinisch Literarische Verlagsgesellschaft, Uelzen

21. Marstedt G, Moebu S (2002) Inanspruchnahme alternativer Methoden in der Medizin. Robert Koch-Institut, Berlin

22. Vickers A, Goyal N, Harland R et al (1998) Do certain countries produce only positive results? A systematic review of controlled trials. Control Clin Trials 19:159-166

23. Cardini F, Wade C, Regalia AL et al (2006) Clinical research in traditional medicine: priorities and methods. Complement Ther Med 14:282-287

24. Fonnebo V, Grimsgaard S, Walach H et al (2007) Researching complementary and alternative treatments-the gatekeepers are not at home. BMCMed Res Methodol 7:7

25. Fischer F, Lewith G, Witt CM et al (2014) A research roadmap for complementary and alternative medicine-what we need to know by 2020 . Forsch Komplementmed 21:e1-e16

26. MacPherson $\mathrm{H}$, Maschino $A C$, Lewith $\mathrm{G}$ et al (2013) Characteristics of acupuncture treatment associated with outcome: an individual patient meta-analysis of 17,922 patients with chronic pain in randomised controlled trials. PLoSOne 8:e77438

27. Vickers AJ, Vertosick EA, Lewith $G$ et al (2018) Acupuncture for chronic pain: update of an individual patient data meta-analysis. J Pain 19:455-474

28. Berman BM, Langevin HM, Witt CM et al (2010) Acupuncture for chronic low back pain. N Engl J Med 363:454-461

29. Qaseem A, Wilt TJ, McLean RM et al (2017) Noninvasive treatments for acute, subacute, and chronic low back pain: a clinical practice guideline from the American College of Physicians. Ann Intern Med 166:514-530

30. AWMF (2011) Nationale VersorgungsLeitlinie Kreuzschmerz. https://www.awmf.org/leitlinien/ detail/II/nvl-007.html.Zugegriffen: 17. Jan. 2020

31. Ratcliffe J, Thomas KJ, MacPherson H et al (2006) A randomised controlled trial of acupuncture care for persistent low back pain: cost effectiveness analysis. BMJ 333:626

32. Witt CM, Reinhold T, Jena S et al (2009) Costeffectiveness of acupuncture in women and men with allergic rhinitis: a randomized controlled study in usual care. Am JEpidemiol 169:562-571
33. ReinholdT, WittCM, Jena Set al (2008) Quality of life and cost-effectiveness of acupuncture treatment in patients with osteoarthritis pain. Eur J Health Econ 9:209-219

34. Ambrosio EM, Bloor K, Macpherson H (2012) Costs and consequences of acupuncture as a treatment for chronic pain: a systematic review of economic evaluations conducted alongside randomised controlled trials. Complement Ther Med 20:364-374

35. Linde K, Niemann K, Schneider A et al (2010) How large are the nonspecific effects of acupuncture? A meta-analysis of randomized controlled trials. BMCMed 8:75

36. Kaptchuk TJ, Kelley JM, Conboy LA et al (2008) Components of placebo effect: randomised controlled trial in patients with irritable bowel syndrome. BMJ 336:999-1003

37. Linde K, Witt CM, Streng A et al (2007) The impact of patient expectations on outcomes in four randomized controlled trials of acupuncture in patients with chronic pain. Pain 128:264-271

38. Krouse JH, Krouse HJ (1999) Patient use of traditional and complementary therapies in treating rhinosinusitis before consulting an otolaryngologist. Laryngoscope 109:1223-1227

39. Roberts J, Huissoon A, Dretzke J et al (2008) A systematic review of the clinical effectiveness of acupuncture for allergic rhinitis. BMC Complement Altern Med 8:13

40. Brinkhaus B, Witt CM, Jena S et al (2008) Acupuncture in patients with allergic rhinitis: a pragmatic randomized trial. Ann Allergy Asthma Immunol 101:535-543

41. Brinkhaus B, Ortiz M, Witt CM et al (2013) Acupuncture in patients with seasonal allergic rhinitis: a randomized trial. Ann Intern Med 158:225-234

42. Xue CC, Zhang AL, Zhang CS et al (2015) Acupuncture for seasonal allergic rhinitis: a randomized controlled trial. Ann Allergy Asthma Immunol 115:317-324.e311

43. Choi SM, Park JE, Li SS et al (2013) A multicenter randomized, controlled trial testing the effects of acupuncture on allergic rhinitis. Allergy 68:365-374

44. Feng S, Han M, Fan Y et al (2015) Acupuncture for the treatment of allergic rhinitis: a systematic review and meta-analysis. Am J Rhinol Allergy 29:57-62

45. Seidman MD, Gurgel RK, Lin SY et al (2015) Clinical practice guideline: allergic rhinitis executive summary. Otolaryngol Head Neck Surg 152:197-206

46. Biedermann T, Kuna P, Panzner P et al (2019) The SQ tree SLIT-tablet is highly effective and well tolerated: results from a randomized, doubleblind, placebo-controlled phase III trial. J Allergy Clin Immunol 143:1058-1066.e1056

47. Reinhold T, Roll S, Willich SN, Ortiz M, Witt CM, Brinkhaus B (2013) Cost-effectiveness for acupunc ture in seasonal allergic rhinitis: economic results of the ACUSAR trial. Ann Allergy Asthma Immunol 111:56-63

48. McDonald JL, Cripps AW, Smith PK et al (2013) The anti-inflammatory effects of acupuncture and their relevance to allergic rhinitis: a narrative review and proposed model. Evid Based Complement Alternat Med 2013:591796

49. McDonald J, Janz S (2017) The acupuncture evidence project: a comparative literature review. AACMA, Brisbane

50. Stener-Victorin E, Zhang H, Li R et al (2019) Acupuncture or metformin to improve insu- lin resistance in women with polycystic ovary syndrome: study protocol of a combined multinational cross sectional case-control study and a randomised controlled trial. BMJ Open 9:e24733

51. Liu Z, Yan S, Wu J et al (2016) Acupuncture for chronic severe functional constipation: a randomized trial. Ann Intern Med 165:761-769

52. Liu Z, Liu Y, Liu B (2017) Acupuncture for stress urinary incontinence-reply. JAMA 318:1500

53. Liu Z, Ai Y, Wang W et al (2018) Acupuncture for symptoms in menopause transition: a randomized controlled trial. Am J Obstet Gynecol 219:373.e371-373.e310

54. Zhao L, Li D, Zheng H et al (2019) Acupuncture as adjunctive therapy for chronic stable angina: a randomized clinical trial. JAMA Intern Med 179(10):1388-1397

55. Pach D, Brinkhaus B, Willich SN (2009) Moxa sticks: thermal properties and possible implications for clinical trials. Complement Ther Med 17:243-246

56. Cardini F, Weixin H (1998) Moxibustion for correction of breech presentation: a randomized controlled trial. JAMA 280:1580-1584

57. Vas J, Aranda-Regules JM, Modesto $M$ et al (2013) Using moxibustion in primary healthcare to correct non-vertex presentation: a multicentre randomised controlled trial. Acupunct Med 31:31-38

58. Zhang M, Qiu L, Zhang J (2007) Observation on therapeutic effect of surround needling plus surround moxibustion on herpeszoster.Zhongguo Zhen Jiu 27:123-125

59. Joos S, Brinkhaus B, Maluche C et al (2004) Acupuncture and moxibustion in the treatment of active Crohn's disease: a randomized controlled study. Digestion 69:131-139

60. MacPherson $\mathrm{H}$, Scullion A, Thomas KJ et al (2004) Patient reports of adverse events associated with acupuncture treatment: a prospective national survey. Qual Saf Health Care 13:349-355

61. Melchart D, Weidenhammer W, Streng A et al (2004) Prospektive investigation of adverse effects of acupuncture in 97733 patients. Arch Intern Med 164:104-105

62. Witt CM, Pach D, Brinkhaus B et al (2009) Safety of acupuncture: results of a prospective observational study with 229,230 patients and introduction of a medical information and consent form. Forsch Komplementmed 16:91-97

63. Cummings M (2009) Myofascial trigger points: Does recent research gives new insights into the pathophysiology? Acupunct Med 27:148-149

64. Manheimer E, Linde K, Lao L et al (2007) Metaanalysis: acupuncture for osteoarthritis of the knee. Ann Intern Med 146:868-877

65. Linde K, Allais G, Brinkhaus B et al (2016) Acupuncture for the prevention of episodic migraine. Cochrane Database Syst Rev 6:CD1218

66. Linde K, Allais G, Brinkhaus B et al (2016) Acupuncture for the prevention of tension-type headache. Cochrane Database Syst Rev 4:CD7587

67. Furlan AD, Van Tulder MW, Cherkin DC et al (2005) Acupuncture and dry-needling for low back pain. Cochrane Database Syst Rev 1:CD1351

68. Trinh K, Graham N, Irnich D et al (2016) WITHDRAWN: Acupuncture for neck disorders. Cochrane Database Syst Rev 11:CD4870

69. Manheimer E, Cheng K, Wieland LS et al (2012) Acupuncture for treatment of irritable bowel syndrome. Cochrane Database Syst Rev 5:CD5111

70. Deare JC, Zheng Z, Xue CCetal (2013) Acupuncture for treating fibromyalgia. Cochrane Database Syst Rev 5:CD7070 
71. Smith CA, Armour M, Zhu X et al (2016) Acupuncture for dysmenorrhoea. Cochrane Database Syst Rev 4:CD7854

72. Manheimer E, Cheng K, Wieland LS et al (2018) Acupuncture for hip osteoarthritis. Cochrane Database Syst Rev 5:CD13010 